\title{
Die Seele des Glaubens
}

Wittgensteins >Philosophische Untersuchungen Themen, die Sprache und die Seele. Wenn man will, kann man sie darum zwei Gebieten der Philosophie zuordnen, der Philosophie der Sprache und der Philosophie der Psychologie. Letzteres, das Interesse an der Seele und an den begrifflichen Grundlagen der Psychologie, ist eines der Hauptthemen von Wittgensteins Philosophieren überhaupt. Seine Überlegungen zum Verhältnis von Innen und Aussen, seelischem Erleben und Ausdruck, Leib und Seele sowie zur Methode der philosophischen Psychologie, aber auch seine detaillierten Einzelerkundungen zu psychologischen Begriffen wie Schmerz, Empfindung, Denken, Vorstellung, Wollen, Selbstbewusstsein usw. haben sich auch nach der Fertigstellung des ersten Teils der ,Untersuchungen in einer Vielzahl von Manuskripten niedergeschlagen.

Methodisch zusammengehalten sind diese begrifflichen Untersuchungen von einer Doppelbewegung: Zum einen zeigt Wittgenstein, wie uns die Formen unserer Sprache (zusammen mit unserem metaphysischen Wunsch nach philosophischen Erklärungen und Theorien) zu problematischen Bildern unserer Seele verführen und wie leer diese Bilder sind. Zum andern helfen uns seine hermeneutisch-grammatischen Erkundungsgänge durch die Verhältnisse unserer psychologischen Begriffe verstehen, wie uns unsere metaphysischen Phantasien daran gehindert haben, die eigentümliche und überaus komplexe Grammatik des Seelischen zu sehen. Das Durcharbeiten dieser Phantasien macht uns bewusst, dass wir die Antwort auf die Frage nach dem Wesen der Seele am falschen Ort gesucht haben, indem wir unser inneres Auger auf das gerichtet haben, was verborgen in unserem Innen geschehen muss, statt hinzuschauen und $\mathrm{zu}$ sehen, wie unser gemeinsames Leben mit den andern den wesentlichen Zusammenhang unserer psychologischen Begriffe darstellt:

"Schmerz liegt so in unserem Leben drin, hat solche Zusammenhänge. (D.h.: nur was so in unserem Leben drinliegt, solche Zusammenhänge hat, nennen wir >Schmerz‘.)«(Z 533).

"Nicht, was Vorstellungen sind, oder was da geschieht, wenn man sich etwas vorstellt, muß man fragen, sondern: wie das Wort /Vorstellung` gebraucht wird. Das heißt aber nicht, daß ich nur von Worten reden will. Denn soweit in meiner Frage vom Wort >Vorstellung die Rede ist, ist sie's auch in der Frage nach dem Wesen derVorstellung. Und ich sage nur, daß diese Frage nicht durch ein Zeigen - weder für den Vorstellenden, noch für den Andern, zu erklären ist; noch durch die Beschreibung irgend ei- 
nes Vorgangs. Nach einer Worterklärung fragt auch die erste Frage; aber sie lenkt unsere Erwartung auf eine falsche Art der Antwort» (PU 370).

"Die große Schwierigkeit ist hier, die Sache nicht so darzustellen, als könne man etwas nicht. Als wäre da wohl ein Gegenstand, von dem ich die Beschreibung abziehe, aber wäre nicht im Stande, ihn jemandem zu zeigen" (PU 374).

Die beiden letzten Zitate reflektieren bereits Wittgensteins Auseinandersetzung mit dem Einwand, dass seine Methode nicht nur, aber gerade auch im Bereich des Seelischen eine Art 'grammatischen Reduktionismus darstelle: Redet Wittgensteins grammatische Methode nur von Worten? Und verneint er mit seiner Kritik an der philosophisch-psychologischen Methode der Introspektion nicht letztlich doch die Eigenständigkeit und Autorität der Erste-Person-Perspektive und damit unser unmittelbares Bewusstsein von unseren Absichten, Hoffnungen, Ängsten usw.? Damit bin ich schon mitten bei dem angelangt, worum es mir in den folgenden Überlegungen geht. Ich zeige in einem ersten Schritt, wie eine verbreitete WittgensteinDeutung tatsächlich den Eindruck erwecken kann, Wittgenstein wolle im Bereich des Seelischen etwas leugnen, er sei eine Art Behaviorist. Ein zweiter Schritt demonstriert an zwei Beispielen (einem aus der Philosophie und einem aus der Theologie), zu welchen Reaktionen diese Missdeutung von Wittgensteins Philosophie (der Psychologie) verführen kann. In einem dritten Schritt versuche ich exemplarisch zu skizzieren, wie eine nicht-reduktive Erkundung des Seelischen nach Wittgenstein aussieht. Ein letzter Schritt gibt einige Hinweise, inwiefern diese Methode auch für die neu erwachte Diskussion um den Seelenbegriff in der Theologie interessant sein könnte.

\section{Die Gefahr einer grammatisch-reduktiven Lesart von Wittgensteins Subjektivitätskritik}

In Erinnerung geblieben sind mir auch die Inhalte einiger Reaktionen. Lebendiger vor Augen steht mir aber noch die Vehemenz, mit der die Kritik an Hans-Peter Großhans'Text /Kritik der Subjektivität durch Ludwig Wittgenstein $\iota^{1}$ vorgetragen wurde. Dass diese vehemente Kritik im Rahmen einer Tagung mit dem Titel /Krise der Subjektivität - und die Antworten darauf` (und dabei innerhalb ei-

${ }^{1}$ Erscheint unter dem Titel `Das moderne Selbstverständnis des Protestantismus und Wittgensteins Kritik der Subjektivität, in: Dalferth, I.U./ Stoellger, Ph. (Hg.), Krisen der Subjektivität. Problemfelder eines strittigen Paradigmas, 63-77. 


\section{Andreas Hunziker}

ner ganzen Reihe von kritischen Beiträgen zur Denkform Subjektivität und ihren theologischen Gebrauchsformen) laut wurde, lässt vermuten, dass es nicht einfach die Kritik als solche an der Denkform Subjektivität war, welche provozierte. Eher dürfte dies eine Reaktion auf die konkrete Weise gewesen sein, wie Wittgenstein an dieser Denkform Kritik übt. Allerdings, reagiert wurde dabei zuerst einmal auf Großhans' Darstellung von Wittgensteins Kritik an der Denkform Subjektivität.

Ich stimme dieser Wittgenstein-Darstellung in ihrer allgemeinen Stossrichtung zu. Wittgensteins späteres Philosophieren ist tatsächlich als eine radikale Kritik an der (metaphysischen) Denkform Subjektivität und deren Bild des - sich autonom konstituierenden, mentalistischen und (infallibel) selbsttransparenten - Selbst lesbar. Und Großhans' Diagnose trifft zu, dass mit dieser Kritik zugleich das moderne Selbstverständnis des Protestantismus in Frage gestellt ist, sofern dies in seinem Religions-, Glaubens- und Theologieverständnis von eben dieser mentalistisch-individualistischen Konzeption des menschlichen Geistes bestimmt ist: Der moderne Protestantismus verstehe sich nämlich - wie Großhans anhand einiger Zitate von Georg Pfleiderer zeigt - als >Religion autonom-selbstgesteuerter, vernünftig-selbstdurchsichtiger, individueller Subjektivität‘; Glaube ist der >religiös-sittliche Vollzug der Selbstkonstituierung individueller Subjektivität; und Theologie macht die religiös-theologische Subjektivität sich selbst durchsichtig. Damit aber werde Religion bzw. Glaube nach dem Modell subjektiv-innerlicher Bewusstseinsvollzüge gedacht, die in der Praxis der Frömmigkeit unmittelbar zum Ausdruck kommen und in der Theologie sprachlich reflektiert und somit objektiviert werden. Es werde also ein von seinem sprachlichen Ausdruck und seiner reflexiven Gestalt unterschiedenes subjektives religiöses Bewusstsein angenommen.

Doch scheint mir, gerade um Großhans' Argumentation in dieser allgemeinen Stossrichtung zu untermauern, in einigen Punkten eine Korrektur seiner Wittgenstein-Darstellung angebracht. Seine Deutung kommt nämlich dem - oben so genannten und in der Literatur zu Wittgenstein weit verbreiteten - 'grammatischen Reduktionismus` zumindest gefährlich nahe: Sofern sich Großhans dem Verdacht nicht ganz entziehen kann, die Überwindung der Denkform Subjektivität um den Preis der Unterdrückung alltäglicher Erfahrungen zu erkaufen (Erfahrungen, die mit dem Sinn dafür zu tun haben, dass ich mich in bestimmter Weise intimer kenne als ich die anderen Menschen kenne und als diese mich kennen), kann er den Eindruck erwecken, Wittgenstein wolle etwas leugnen (PU 304f), als sei dieser etwa eine Art Behaviorist (PU 307). Und er macht damit zugleich 
wiederum diejenigen metaphysischen Stimmen stark, gegen die seine Überlegungen gerichtet sind: "Vielleicht hilft die grammatische Methode Wittgensteins ja tatsächlich unsere sprachlich erschlossene, äussere Welt zu verstehen. Aber für mein Inneres, für meine Absichten, Hoffnungen und Ängste braucht es das feinere Instrument der inneren Beobachtung. Woher, wenn nicht durch eine solche Introspektion, habe ich nämlich mein Wissen, ob ich im Moment gerade Schmerz empfinde und wie es für mich ist, diesen Schmerz zu empfinden? Schmerz ist nicht einfach Sprache und darum der grammatischen Methode unzugänglich".

Wie eine andere, nicht-reduktive Wittgenstein-Deutung auszusehen hätte, die uns nicht mehr das Gefühl gibt, Wittgenstein wolle im Bereich des Seelischen etwas leugnen oder zumindest eine Grenze zwischen dem sprachlich Darstellbaren und Nicht-Darstellbaren ziehen (so dass wir das Gefühl haben, als könnten wir etwas nicht: als sei da wohl ein seelischer Gegenstand, von dem wir die Beschreibung abziehen, aber wir seien nicht im Stande, ihn jemandem zu zeigen), darauf komme ich im dritten Abschnitt zurück. Jetzt beschränke ich mich auf einige knappe Hinweise, inwiefern Großhans den Eindruck erwecken kann, als bedeute Wittgensteins Kritik an der metaphysischen Denkform Subjektivität auch eine Verabschiedung unserer gewöhnlich-alltäglichen Erfahrung von Subjektivität, nämlich unserer Erfahrung, Menschen mit einem privaten Innen zu sein: "And doesn't Wittgenstein deny privacy? What else ist the teaching of his obsessive emphasis on the publicness of language and on the outwardness of criteria? (Cavell 1979, 329).

- Es ist zumindest missverständlich, wie Großhans aus Wittgensteins Kritik an der Vorstellung von inneren subjektiven Bewusstseinsvollzügen und -zuständen, die in der Sprache abgebildet oder reflektiert werden - mit Walter Schulz! - folgert, dass damit der Gedanke der Subjektivität überhaupt zum Verschwinden gebracht worden sei: Das Verstehen müsse sich auf die Sprache konzentrieren, die eine systematische Ordnung darstelle und die in sich auslegbar sei ohne Rückgriff auf eine sich verstehende Subjektivität. Damit ist ein Gegensatz zwischen Sprache und Subjektivität aufgebaut, der zumindest solange problematisch bleibt, als nicht genauer bestimmt wird, was unter 'Subjektivität und 'Sprache jeweils zu verstehen ist.

- Im Blick auf den Begriff der 'Subjektivität besteht die Gefahr, das Kind (unser Sinn dafür, Menschen mit einem je eigenen, subjektiven Innen zu sein) mit dem Bade (der sprachunabhängigen bedeutungskonstitutiven Subjektivität und deren mentalistischem Individualismus) auszuschütten. 


\section{Andreas Hunziker}

- Und auch der verwendete Begriff der 'Spracher weckt problematische Assoziationen. Sprache ist bei Wittgenstein gerade kein unpersönliches, nach exakten Regeln ablaufendes Kalkül. Vielmehr vollzieht sich unser Sprechen auf dem Hintergrund einer kontingent-naturgeschichtlich bedingten Übereinstimmung in unseren menschlichen Formen des Lebens, die uns unserer subjektiven Verantwortung für diese Übereinstimmung in der Sprache und für die freie Projektion von Worten in neue Kontexte gerade nicht (im Sinne einer apriorisch-metaphysischen Bedeutungsmatrix) abnimmt:

"We learn and teach words in certain contexts, and then we are expected and expect others, to be able to project them into further contexts. Nothing insures that this projection will take place (in particular, not the grasping of universals nor the grasping of books of rules), just as nothing insures that we will make, and understand, the same projections. That on the whole we do is a matter of our sharing routes of interest and feeling, modes of response, senses of humor and of significance and of fulfillment, of what is outrageous, of what is similiar to what else, what a rebuke, what forgiveness, of when an utterance is an assertation, when an appeal, when an explanation - all the whirl of organism Wittgenstein calls iforms of lifer. Human speech and activitiy, sanity and community, rest upon nothing more, but nothing else, than this. It is a vision as simple as it is difficult, and as difficult as it is (and because it is) terrifying. To attempt the work of showing its simplicity would be a real step in making available Wittgenstein's later philosophy“ (Cavell 1976, 52).

Bleiben diese Dimensionen von Wittgensteins Verständnis der Sprache ausgespart, und bleibt überdies der auf unseren sprimitiven Reaktionen aufbauende komplizierte Bildungsprozess unerwähnt, in den wir seit den ersten Anfängen unserer Kindheit involviert sind und der uns in komplizierten Inkulturationsprozessen allererst zu den Menschen mit einem Geist, die wir sind, macht - dann legt folgende Bemerkung von Großhans eine Art grammatischen Reduktionismus doch sehr nahe: "Vielmehr geschieht im Geist des Menschen nichts ohne dieselbe Sprache, die materiell in Erscheinung tritt. Der Geist ist Sprache» (Großhans 2005, 65).

\section{Reaktionen auf Wittgensteins vermeintlichen grammatischen Reduktionismus}

1. Hans Schnädelbach:

Wittgenstein-Deutungen wie diejenige von Großhans sind nicht unschuldig an der Kritik, wie sie Schnädelbach in seinem Aufsatz >Phänomenologie und Sprachanalyse` vorbringt. Wittgenstein werde dem phänomenalen Bewusstsein nicht gerecht, dem 
"Bewußtsein davon, wie es ist, in einem bestimmten Zustand zu sein, eine bestimmte Empfindung zu haben oder ein Gefühl, und von ihm ist ein Wissen, daß ich es bin, der sich in jenem Zustand befindet, ihn erlebt und dabei genau dies empfindet oder fühlt, wohl nicht wegdenkbar. ... Diese Tatsache erklärt, warum die Bewußtseinsphänomenologie auch heute noch überall dort zu überzeugen vermag, wo der grammatische Nominalismus offensichtlich zu kurz greift: bei den Wahrnehmungsurteilen, die Husserls gesamte Philosophie bestimmen, bei den Stimmungen und existentiellen Befindlichkeiten, wo Scheler und Heidegger ihre phänomenologischen Stärken erweisen, und nicht zuletzt im Feld des präreflexiven Selbstbewußtseins, das sich wohl nicht aus der grammatischen Kompetenz des Verfügens über die Personalpronomina allein erkären läßt, sondern dabei offensichtlich schon vorausgesetzt ist" (Schnädelbach 2001, 265f).

Schnädelbach missversteht Wittgenstein zwar gründlich, wenn er ihm eine (im Vergleich mit unserer Alltagserfahrung reduktive) Position unterstellt, die dieser gerade umgekehrt als Resultat eines grammatischen Missverständnisses entlarvt:

"Wenn Wittgenstein das, was saußer dem menschlichen Benehmen` Fiktion sein soll, nun als eine grammatische Fiktion versteht, dann ist er eben grammatischer oder 'logischer Behaviorist, und sieht man auf seine Deutung mentaler Ausdrücke wie smeinen', sdeuten', swiedererkennen<, 'wünschen`, ssich erinnern etc., dann ist der Unterschied zum psychologischen Behaviorismus nicht so groß« (ebd., 260).

Aber Schnädelbach geht immerhin hinter die sprachanalytische Kritik an Husserls mentalistischer Referenztheorie der Bedeutung nicht zurück. Wittgensteins Privatsprachenargument habe den Rückweg in den methodischen Solipsismus einer reinen Phänomenologie versperrt, vom Gebrauch der Sprache im Gegensatz zu etwas anderem - etwa einer 'Schau reiner intentionaler Erlebnisse - können wir nicht sprechen. So läuft Schnädelbachs These eines komplementären sund zwischen Phänomenologie und Sprachanalyse auf eine friedliche Aufteilung der Zuständigkeitsbereiche hinaus: Für die uns sprachlich erschlossene Welt ist die sprachanalytische Philosophie zuständig; doch gibt es Bereiche unseres Erlebens, die sich zwar nicht unabhängig vom Feld der Grammatik befinden, welche die grammatische Analyse aber nicht auszuschöpfen vermag: »das phänomenale Bewußtsein ist ein Komplement unserer sprachlichen Kompetenz« (ebd., 267). Dabei lebt seine These von der Voraussetzung, dass die zukunftsträchtigen Varianten von Phänomenologie (seit Heidegger) und Sprachanalyse (mit Wittgenstein) hermeneutisch geworden sind und sich darum entscheidend angenähert haben. 


\section{Andreas Hunziker}

Hinter die (Sprach-)Kritik an der bedeutungskonstitutiven Subjektivität will also auch Schnädelbach nicht zurück. Allerdings verbaut er sich mit dem Bild, wonach die Sprachanalyse sozusagen fürs Äussere und Grobe, die Phänomenologie fürs Innere und Feine zuständig sein soll, nicht nur ein besseres Verständnis der (zukünftigen) Möglichkeiten des Gesprächs zwischen Phänomenologie und Sprachanalyse. Sondern er missversteht damit auch die methodische Eigenart von Wittgensteins hermeneutisch-grammatischen Erkundungsgängen durch die Verhältnisse unserer psychologischen Begriffe und damit zugleich einen wichtigen Teil der eigentümlichen und überaus komplexen Grammatik des Seelischen.

\section{Ulrich Barth:}

Dessen Aufsatz >Selbstbewußtsein und Seele gehört in eine ganze Reihe neuerer theologischer Publikationen, die das wiedererwachte Interesse am Begriff der Seele ausdrücken (nach Barth ist der Seelenbegriff in der Theologie, Philosophie und Psychologie des 20. Jahrhunderts - als Resultat der Destruktion seiner metaphysischen Grundlage am Ende des 18. Jahrhunderts - weitgehend vernachlässigt worden). Dabei fällt auf, dass sich Barths Seelenverständnis mit dem eng berührt, was Schnädelbach als phänomenales Bewusstsein beschreibt und diesem zufolge in den Zuständigkeitsbereich nicht der Sprachanalyse, sondern der Bewusstseinsphänomenologie in der Tradition Husserls fällt:

"Die Phänomene, die wir unter dem Begriff der Seele zusammenfassen, betreffen also vornehmlich die in der Innenperspektive zugänglichen Erlebnisse eines Menschen« (Barth 2004, 209).

"Die Seele des Menschen ist das durch den Leibbezug gestiftete offene Ganze seiner psychophysischen Zustände im Wie ihres inneren Erlebens" (ebd., 215; Hervorhebung von mir).

Barth will in seinen Überlegungen vor allem zeigen, dass die Philosophie der Subjektivität nicht als Gegenmodell zu einem solchen Begriff der Seele verstanden werden muss. Ein Durchgang von Kant über Husserl, Heidegger, Gehlen und die Gestaltpsychologie bis hin zur gegenwärtigen empirischen Emotionspsychologie soll bei der Bewältigung dieser Aufgabe helfen. Dazu unterscheidet er zuerst mit Kant zwischen dem reinen Selbstbewusstsein des Denkens und der in den Bereich des inneren Sinns gehörenden empirischen Erscheinung der Seele. Daran lässt sich dann Husserl anschliessen,

"weil auch er (1) im transzendentalen Cogito das Fundament der Philosophie als Wissenschaft erblickte, (2) den methodischen Status der Psychologie von der Transzendentalphilosophie her bestimmte, (3) den Begriff 
der Seele für unverzichtbar hielt und (4) in der Selbstwahrnehmung den Ansatzpunkt aller psychologischen Forschung gegeben sah« (ebd., 203f).

Husserls transzendentalphilosophisch fundierte empirische Psychologie der Seele beziehe damit eine Position jenseits von physikalistischem Naturalismus auf der einen (Husserl halte an der verstehenden Methode und einem pointiert mentalistischen Seelenbegriff fest) und psychophysischem Dualismus auf der anderen Seite (lebensweltlich sei Psychisches und Körperliches immer schon miteinander verschränkt). Dabei werde mit letzterem der Leibbezug für das Seelenverständnis fundamental, indem dieser zum (empirisch-vorreflexiven) Einheitsgaranten all unserer psychophysischen Erlebnisse werde. Damit sieht Barth zugleich die Lösung gegeben auf das sich im Durchgang durch Heidegger, Gehlen, Gestaltpsychologie und Emotionspsychologie ergebende Desiderat eines Einheitsgaranten, der die von der psychologischen Forschung aufgewiesene emotionelle Vereinheitlichung von Erlebnissen durch die Dominanz einer Stimmung überhaupt ermöglicht:

»Die im Zumutesein sich artikulierende Zuständlichkeit einer Person, die wir als Seele bezeichneten, repräsentiert deshalb einen psychischen Gesamtzustand, weil die von jener Gestimmtheit emotional qualifizierten Erlebnisse mit dieser zusammen auf einen identischen Leib bezogen sind. Insofern kann man die Seele des Menschen als eine vorreflexive Gestalt von Identität bezeichnen" (ebd., 215).

Theologisch teile ich Barths Interesse am Begriff der Seele. Und philosophisch plädiere auch ich nicht für eine Wiederaufnahme des substanzontologischen Seelengedankens. Vielmehr sehe ich die gegenwärtige Herausforderung ganz ähnlich wie Barth darin, diesseits von einem (starken) Naturalismus auf der einen und einem psychophysischen Dualismus auf der anderen Seite die lebensweltliche Verschränkung von Psychischem und Körperlichem zu beschreiben. Trotzdem dürften Barths Überlegungen zum Begriff der Seele über die kleine Gruppe derjenigen hinaus, für welche eine Variante der Subjektivitätsmetaphysik das organisierende Zentrum ihres philosophisch-theologischen Denkens darstellt, kaum wegweisend werden. Denn:

- Die Weise, wie Barth versucht, den Begriff der Seele als Ergänzung zum Prinzip Selbstbewusstsein herauszustellen, ist mit seiner eigenen methodischen Maxime, wonach alles darauf ankomme, "ob es gelingt, von empirisch gesättigten und empirisch bewährten Einzelbeschreibungen überzugehen zu strukturellen Aussagen höherer Ordnung" (ebd., 216), nur schwerlich vereinbar. Eher scheint er umgekehrt in seinem philosophie- und psychologiegeschichtlichen Durchgang diejenigen Befunde auszuwählen und zu einem Ganzen 


\section{Andreas Hunziker}

zusammenzufügen, die in seinen subjektivitätstheoretischen Begriffsrahmen möglichst nahtlos (bzw. ohne an ihm Schaden anzurichten) hineinpassen: Husserl wird zum »einzigen Fachphilosophen des 20. Jahrhunderts, der das Problem noch einmal in seiner gesamten Komplexität aufrollte« (das Problem besteht snatürlich darin, Transzendentalphilosophie und empirische Psychologie der Seele miteinander zu vermitteln); Heidegger wird für seinen Antimentalismus kritisiert, dafür aber wird die mit seinem Stimmungsbegriff verbundene Ganzheitserfahrung positiv aufgenommen; mit der ausschliesslichen Verortung der Seelenproblematik im Bereich der empirischen Psychologie lässt sich nahtlos an Kant und Husserl (und das individualistisch-mentalistische Bild des Geistes in der gegenwärtigen Kognitionswissenschaft?) anschliessen.

- Zwar meint auch Schnädelbach, dass im Bereich des Wie unseres Erlebens Husserls Phänomenologie der Wahrnehmungsurteile, Heideggers Hermeneutik der Stimmungen und existentiellen Befindlichkeiten (und Franks Beschreibungen des präreflexiven Selbstbewusstseins) ihre besondere Stärke zeigen. Und auch wenn bei ihm klärungsbedürftig bleibt, was es heisst, dass das phänomenale Bewusstsein ein Bereich ist, den die grammatische Analyse snicht auszuschöpfen vermag`, dieser Bereich aber doch snicht unabhängig vom Feld der Grammatik bestehe - klar ist immerhin, dass er nicht ein von seinem sprachlichen Ausdruck gänzlich unabhängiges Bewusstsein oder Erleben annehmen will. Dies allerdings ist bei Barth gerade nicht mehr klar, wenn er (mit Husserl) die Introspektion als die Methode der Psychologie der Seele behauptet und als das Entscheidende der Introspektion folgendes betrachtet:

"Der Vollzug von Selbstwahrnehmung impliziert als solcher noch nicht den Akt sprachlicher Selbstzuschreibung und ist darum von der Frage der Wahrheitsfähigkeit oder Verifizierbarkeit derselben nicht tangiert. In psychologisch-methodischer Hinsicht geht es bei Introspektion vielmehr in erster Linie um die Frage der hermeneutischen Zugänglichkeit innerer Erlebnisse, ihres intentionalen Gehalts wie der sie begleitenden Überzeugungen" (ebd., 208).

Was meint Barth damit? Geht es ihm um einen vorsprachlichen im Sinne von vorbegrifflichen Zugang zu einem mental Gegebenen? Eine solche Mixtur von Mentalismus und empiristischem Mythos des Gegebenen scheint jedenfalls in seinem Rekurs auf Gehlen vorausgesetzt:

"Sprachliche Bezeichnungen heften sich aber nicht unmittelbar an äußere Gegenstände, sondern an deren empirisch gewachsene Erinnerungsphantasmen, die jene allererst mental aufrufbar und beliebig verfügbar machen. Die Sprache ist also ein Symbolsystem zweiter Stufe. Die elementa- 
re symbolische Ideation vollzieht sich im Übergang von körperlichen Sinneseindrücken zu bewußten Vorstellungsbildern, die aber an jene genetisch zurückgebunden sind. Das besagt: Die Seele als das Reich unserer Vorstellungen ist zunächst nichts anderes als eine sinnere Außenwelt«" (ebd., 207).

Barth bejaht an dieser Stelle also genau, was Wittgensteins Privatsprachenargument (unter anderem) kritisiert (vgl. Großhans 2005, 70).

- Das eben Gesagte ist charakteristisch für die Weise, wie Barth mit »den sprachphilosophischen Debattenzusammenhängen - von Wittgenstein bis Tugendhat" umgeht, in denen "wiederholt Kritik geübt [wurde] sowohl an der Leistungskraft von Introspektion als auch an der Tauglichkeit des Innen/Außen-Schemas überhaupt « (ebd., 208). Der sprachanalytischen Kritik am Innen/Aussen-Schema und der mit diesem verbundenen Vergegenständlichung des Psychischen stimmt er zwar zu. Und auch mit dem Hinweis auf die zentrale Funktion des Begriffs des Ausdrucks von Empfindungen in Wittgensteins Versuch, den Hiatus zwischen Innen und Aussen zu unterlaufen, stellt er etwas bei Wittgenstein ganz Entscheidendes fest. Was aber ist die argumentative Funktion von solchen Zugeständnissen, wenn sie dann in seinen eigenen Argumentationen kaum noch eine konstruktive Rolle spielen bzw. ihnen an anderer Stelle sogar widersprochen wird (niemand »kann in das Innere eines anderen Menschen hineinblicken", ebd., 209)?

- Dazu passt, dass Barth der sprachanalytischen Richtung pauschal die »Bestreitung einer besonderen Erschließungskraft von Introspektion unterstellt« (ebd., 208). Zwar scheint er der sprachanalytischen Kritik insofern Recht zu geben, als sie Descartes'These von der Irrtumsimmunität der Introspektion betrifft. Damit aber (mit dem Verweis auf Russell und Ryle) der gesamten sprachanalytischen Tradition jeglichen affirmativen Gebrauch des Introspektionsbegriffs (im Sinne eines privilegierten Zugangs eines Individuums $\mathrm{zu}$ seinen $\mathrm{Ab}-$ sichten, Ängsten, Wünschen, Emotionen, Stimmungen usw.) abzusprechen, weil diese (vermeintlich) nicht bemerkt hat, dass es auch eine zwar fallible, aber für eine verstehende Psychologie zentrale Innenperspektive gibt - dies trifft z.B. auf Wittgenstein gerade nicht zu. Damit ist aber in einer gegenüber Schnädelbach noch zugespitzten Weise die Sprachanalyse von vorneherein als für den Bereich des Seelischen (im Sinne Barths) irrelevant erklärt.

- Wenn ich sage, dass Wittgenstein nicht die Eigenständigkeit der Erste-Person-Perspektive verneint, dann heisst dies nicht, dass sein Introspektionsverständnis mit demjenigen von Barth deckungsgleich ist. Wittgenstein geht auch nicht wie Barth davon aus, dass die Phä- 


\section{Andreas Hunziker}

nomene, die wir unter dem Begriff der Seele zusammenfassen, vornehmlich die in der Innenperspektive zugänglichen Erlebnisse eines Menschen betreffen. Ja, Wittgensteins Erkundungen von solchen psychologischen Begriffen wie Absicht, Denken, Vorstellung, Emotion, Erinnerung, Bewusstsein usw. operieren normalerweise gerade nicht mit der Methode der Introspektion, sondern fragen nach der Rolle, die ein Begriff in unserem menschlichen Leben spielt (wobei dies im Modus der Selbsterkenntnis und damit sehr wohl in einer Beteiligtenperspektive geschieht). Damit wird die grammatische Methode aber nicht zu einem blossen Reden von Worten, sondern es ist dies eben Wittgensteins Weise, wie er diesseits von einem starken Naturalismus auf der einen und einem psychophysischen Dualismus auf der anderen Seite die lebensweltliche Verschränkung von Psychischem und Körperlichem bzw. von Innen und Aussen zu beschreiben vermag: "Der menschliche Körper ist das beste Bild der menschlichen Seele" (PU II, iv, 496).

Dies ist gerade die Pointe von Wittgensteins Methode, dass sie das Wesen der Seele nicht in irgendwelchen Prozessen oder $\mathrm{Zu}-$ ständen im abstrakten Innen des menschlichen Geistes (oder Gehirns) sucht, indem wir unser inneres Auge (bzw. die empirischen Methoden zur Untersuchung der neuronalen Prozesse) auf das richten, was verborgen in unserem Innen geschehen muss, sondern dass sie uns auffordert, hinzuschauen und zu sehen, wie unser Leben mit den andern den wesentlichen Zusammenhang unserer psychologischen Sprachspiele darstellt. Und es ist dies die Weise, wie Wittgenstein zu einer Differenziertheit und Komplexität im Verstehen dieser Phänomene kommt, von der ich bei Barth nur wenig sehe. Oder ist es z.B. nur dem besonderen Argumentationskontext geschuldet, wenn Barth zwar feststellt, dass zum Erlebnisfeld der Innenperspektive "eine Vielzahl unterschiedlicher psychischer Funktionen" gehört: "Perzeptionen, Kognitionen, Emotionen, Motivationen, Volitionen und manches andere mehr« (ebd., 209), dann aber nur die Emotionen weiter untersucht und diese wiederum mit den Stimmungen gleichsetzt, um so (über den Leibbezug) die Seele als zur Einheit gebrachten Gesamterlebniszustand einer Person beschreiben zu können?

Vielleicht läuft Barths Unterfangen, den Begriff Seele als Ergänzung zum Prinzip Selbstbewusstsein unter heutigen Theoriebedingungen zu exponieren, letztlich auf nicht viel mehr als auf den (m.E. nicht besonders erfolgreichen) Versuch hinaus, den subjektivitätstheoretischen Knochen um ein bisschen seelisch- 
emotionales Fleisch anzureichern. Und stellt die ausschliessliche Verortung der Seele im Bereich des Empirischen faktisch nicht eine Art Immunisierung gegenüber einer philosophischen Psychologie dar, die es gar nicht für nötig hält, hinter dem überaus komplexen und in sich sehr unterschiedlichen Phänomenbereich des Seelischen noch einmal nach einer (begründenden) Struktur sSubjektivität zu suchen?

Mit der richtigen Erkenntnis, dass mit einer grammatisch-reduktiven Deutung von Wittgensteins Philosophieren etwas geleugnet wird, was wesentlich zu unserem menschlichen Erleben gehört, ist jedenfalls noch nicht die Frage beantwortet, in welcher Terminologie und mit welcher Methode dieses innere Erleben beschrieben werden soll.

\section{Wittgensteins nicht-reduktive Erkundungen der Begriffsverhältnisse der Seele}

Wittgensteins Sprachphänomenologie widerstreitet nicht unserem Sinn dafür, Menschen mit je eigenem Innenleben, mit Geist und Individualität zu sein, versucht diese Phänomene aber konsequent als Teil unseres gewöhnlichen Lebens zu verstehen. Pointiert gesagt: Von Wittgenstein können wir lernen, wie wir nicht-reduktiv und nicht-metaphysisch zugleich über die Seele sprechen können. Ich versuche, dies an zwei Beispielen exemplarisch zu skizzieren.

\section{Der Ort des Denkens:}

Das erste Beispiel zeigt uns eine Weise (unter vielen anderen), wie uns die Oberflächengrammatik unserer Sprache dazu verführen kann, uns ein falsches Bild des Geistigen bzw. Seelischen als etwas innerhalb unseres Körpers Verborgenen zu machen:

"Vielleicht ist der Hauptgrund, aus dem wir so stark dazu neigen, vom Kopf als dem Ort unserer Gedanken zu sprechen, dieser: das Bestehen der Wörter `Denken Tätigkeiten bezeichnen, wie Schreiben, Sprechen etc., läßt uns nach einer Tätigkeit suchen, die von diesen verschieden und doch analog zu ihnen ist und die dem Wort /Denken entspricht. ... Und nun, da die Sätze irgendwo sind, suchen wir nach einem Ort für den Gedanken. ... Wir sagen: 'Sicher ist der Gedanke etwas; er ist nicht nichts`, und alles, was man darauf antworten kann, ist, daß das Wort 'Gedanker seinen Gebrauch hat, der von völlig anderer Art ist als der Gebrauch des Wortes `Satz« (Blaues Buch, 23f).

Weitere solche möglichen Fehlerquellen sind z.B. (vgl. McGinn 1993, 698f): Wir deuten die Unsicherheit, welche mit der Unbe- 


\section{Andreas Hunziker}

stimmtheit zu tun hat, die zu unseren psychologischen Sprachspielen wesentlich gehört, als ein Zeichen der Verborgenheit der geistigen Zustände des anderen; dasselbe tun wir mit der Fähigkeit von anderen, ihre Gefühle und Gedanken vor uns zu verbergen; wir deuten, die Erste-Person/Dritte-Person-Asymmetrie, die unsere psychologischen Begriffe charakterisiert, in der Terminologie eines Subjekts, dass ein Wissen von einem inneren Bereich hat, dessen Inhalte die anderen nur erraten können usw.

\section{Ausdruck und Inneres²:}

"Wenn ich von mir selbst sage, ich wisse nur vom eigenen Fall, was das Wort `Schmerz bedeutet, - muß ich das nicht auch von den Andern sagen? Und wie kann ich denn den einen Fall in so unverantwortlicher Weise verallgemeinern?

Nun, ein Jeder sagt es mir von sich, er wisse nur von sich selbst, was Schmerzen seien! - Angenommen, es hätte Jeder eine Schachtel, darin wäre etwas, was wir 'Käferı nennen. Niemand kann je in die Schachtel des Andern schaun; und Jeder sagt, er wisse nur vom Anblick seines Käfers, was ein Käfer ist. - Da könnte es ja sein, daß Jeder ein anderes Ding in seiner Schachtel hätte. Ja, man könnte sich vorstellen, daß sich ein solches Ding fortwährend veränderte. - Aber wenn nun das Wort 'Käfer dieser Leute doch einen Gebrauch hätte? - So wäre er nicht der der Bezeichnung eines Dings. Das Ding in der Schachtel gehört überhaupt nicht zum Sprachspiel; auch nicht einmal als ein Etwas: denn die Schachtel könnte auch leer sein. - Nein, durch dieses Ding in der Schachtel kann ggekürzt werden'; es hebt sich weg, was immer es ist.

Das heißt: Wenn man die Grammatik des Ausdrucks der Empfindung nach dem Muster von `Gegenstand und Bezeichnung` konstruiert, dann fällt der Gegenstand als irrelevant aus der Betrachtung heraus« (PU 293).

")Und doch gelangst du immer wieder zum Ergebnis, die Empfindung selbst sei ein Nichts. $<$ Nicht doch. Sie ist kein Etwas, aber auch nicht ein Nichts! Das Ergebnis war nur, daß ein Nichts die gleichen Dienste täte, wie ein Etwas, worüber sich nichts aussagen läßt. Wir verwarfen nur die Grammatik, die sich uns hier aufdrängen will« (PU 304).

Es sind nicht zuletzt solche Bemerkungen, die Schnädelbach annehmen lassen, Wittgenstein vertrete eine Art grammatischen Reduktionismus, er verneine, dass etwas in unserem Inneren vorgeht oder suggeriere zumindest, dieses etwas sei in unserer sprachlich-öffentlichen Kommunikation unerheblich. Dabei will Wittgenstein vielmehr in der charakteristischen Doppelbewegung seines Denkens zeigen, wie uns ein bestimmtes Bild vom Innen und Aussen (der

\footnotetext{
${ }^{2}$ Ich referiere in diesem Abschnitt über weite Strecken McGinn 1997,
} $143 \mathrm{ff}$. 
Schmerz ist innen, der Schrei ist aussen) zu leeren metaphysischen Konstruktionen des Seelischen verfuhrt, zu Konstruktionen also, die unseren alltäglichen Gebrauch der psychologischen Begriffe und damit das, was ein Phänomen wie Schmerz oder Erinnerung für uns in unserem alltäglichen Leben ist, gerade nicht zu verstehen erlauben. Das Verstehen dieser Phänomene besteht darum in der Klärung ihrer Grammatik, indem wir hinschauen und sehen, welchen Ort sie in den komplizierten Begriffsverhältnissen unseres menschlichen Lebens tatsächlich haben: "Schmerz liegt so in unserem Leben drin, hat solche Zusammenhänge. (D.h.: nur was so in unserem Leben drinliegt, solche Zusammenhänge hat, nennen wir 'Schmerz‘.)« (Z 533).

$\mathrm{Zu}$ diesem Verstehen des Phänomens Schmerz gehört das Verstehen, wie unser gewöhnlicher Begriff des Schmerzes mit dem natürlichen Ausdruck des Schmerzes im Schmerzverhalten wesentlich zusammenhängt und damit zu einem Gebrauch der Innen/AussenUnterscheidung, in dem unser Ausdrucksverhalten grammatisch zentral ist (darauf weist auch Ulrich Barth hin, aber er führt diesen Gedanken nicht weiter aus):

"Wie beziehen sich Wörter auf Empfindungen? ... Dies ist eine Möglichkeit: Es werden Worte mit dem ursprünglichen, natürlichen, Ausdruck der Empfindung verbunden und an dessen Stelle gesetzt. Ein Kind hat sich verletzt, es schreit; und nun sprechen ihm die Erwachsenen zu und bringen ihm Ausrufe und später Sätze bei. Sie lehren das Kind ein neues Schmerzbenehmen.

'So sagst du also, daß das Wort 'Schmerz eigentlich das Schreien bedeute? - Im Gegenteil; der Wortausdruck des Schmerzes ersetzt das Schreien und beschreibt es nicht« (PU 244).

Nur, so wendet Wittgensteins Gesprächspartner ein, dies läuft darauf hinaus, dass es keinen Schmerz ohne Schmerzbenehmen gibt (PU 281), und dies widerspricht doch offensichtlich unserer alltäglichen Erfahrung. Wittgenstein antwortet darauf:

"Es kommt darauf hinaus: man könne nur vom lebenden Menschen, und was ihm ähnlich ist, (sich ähnlich benimmt) sagen, es habe Empfindungen; es sähe; sei blind; sei taub; sei bei Bewußtsein, oder bewusstlos" (PU 281).

Wittgenstein nimmt also den grammatischen Zusammenhang zwischen dem Schmerz und dem Schmerzverhalten nicht zurück, sondern macht deutlich, dass dieser komplexer ist, als ihm der Gesprächspartner unterstellt, dass dazu eben auch die Möglichkeit der Täuschung, der Unterdrückung des Schmerzbenehmens usw. gehört, vgl. PU II, xi, 576f: 


\section{Andreas Hunziker}

"Verstellung ist natürlich nur ein besonderer Fall davon, daß einer, z.B., eine Schmerzäußerung von sich gibt und nicht Schmerzen hat. Wenn dies überhaupt möglich ist, warum sollte denn dabei immer Verstellung statthaben, - dieses sehr spezielle Muster auf dem Band des Lebens? Ein Kind muß viel lernen, ehe es sich verstellen kann. (Ein Hund kann nicht heucheln, aber er kann auch nicht aufrichtig sein.)《.

Und indem er an diesem grammatischen Zusammenhang festhält, widerspricht er unserer Neigung, das Innen/Aussen-Bild so anzuwenden, dass wir irgendwo tief innerhalb unseres Körpers einen ontologischen Schnitt machen, so dass der Körper zum Bereich der äusseren Dinge gehört, während der Schmerz zum psychischen Bereich gehört, der im Innern unseres Körpers ist, und in dem die bewussten Erfahrungen ablaufen. Denn auch wenn wir diesen inneren Bereich als Seele bezeichnen würden (PU 283), wir würden den menschlichen Körper damit begrifflich gänzlich vom Begriff Schmerz ablösen und wir könnten den Schmerz statt einem Körper dann gerade so gut einem Stein zuschreiben (PU 283).

All dem widerspricht aber nach Wittgenstein der Gebrauch unserer psychologischen Begriffe. Unsere allägliche Grammatik macht eben keinen Schnitt innerhalb des Körpers, sondern zwischen Körpern verschiedener Art:

"Und nun schau auf eine zappelnde Fliege, und sofort ist diese Schwierigkeit verschwunden und der Schmerz scheint hier angreifen zu können ... Und so scheint uns auch ein Leichnam dem Schmerz gänzlich unzugänglich. - Unsre Einstellung zum Lebenden ist nicht die zum Toten. Alle unsre Reaktionen sind verschieden« (PU 284).

Gegen die falsche Verwendung des Innen/Aussen-Bildes im Sinne einer Unterscheidung zwischen einem äusseren-physischen und einem inneren-psychischen Bereich stellt Wittgenstein also die in der Grammatik unserer Lebensformen wurzelnde Unterscheidung zwischen dem Lebenden und dem Nicht-Lebenden heraus. Und so ist auch der Leichnam nicht einfach die eine Hälfte von dem, was vorher als Körper und Geist zusammen war, sondern er wird allererst zu etwas, das dem Schmerz gänzlich unzugänglich ist. Der lebendige Körper umgekehrt ist nicht nur in dem Sinne lebendig, dass er sich im Gegensatz zum Toten bewegt (PU 284), sondern in dem Sinne, dass seine Bewegungen und Gesten eine bestimmte Bedeutung haben. Und wenn Wittgenstein dies mit dem Erkennen des Gesichtsausdrucks vergleicht (PU 284), unserer Fähigkeit, den Anderen unmittelbar als etwas zu lesen bzw. zu sehen, dann sind wir bei einem zentralen Thema von Wittgensteins Philosophie der Psychologie an- 
gelangt, das in der Wendung eine Einstellung zur Seele prägnant ausgedrückt ist:

"Ich glaube, daß er leidet.< - Glaube ich auch, daß er kein Automat ist? Nur mit Widerstreben könnte ich das Wort in diesen beiden Zusammenhängen aussprechen.

(Oder ist es so: ich glaube, daß er leidet; ich bin sicher, daß er kein Automat ist? Unsinn!) ...

IIch glaube, daß er kein Automat ist hat, so ohne weiteres, noch gar keinen Sinn. Meine Einstellung zu ihm ist eine Einstellung zur Seele. Ich habe nicht die Meinung, daß er eine Seele hat....

Der menschliche Körper ist das beste Bild der menschlichen Seele» (PU II, 495f).

Unsere psychologischen Begriffe wie Schmerz, Absicht oder Freude sind mit den Ausdrucksformen der lebendigen Körper von Menschen und anderen Tieren intern verbunden. Unsere psychologischen Sprachspiele zu lernen, das heisst darum nicht, dass wir Prozesse in einem inneren Bereich zu identifizieren lernen (PU 283: "Meine Erziehung hätte mich darauf geführt, indem sie mich auf die Gefühle in mir aufmerksam machte, und nun übertrage ich die Idee auf Objekte außer mir«). Es heisst vielmehr, dass wir lernen, an immer komplexeren Handlungs- und Antwortmustern teilzunehmen und sie auch zu verstehen. Denn ein psychologischer Begriff wie Schmerz hängt mit bestimmten Mustern in der komplizierten Lebensform von lebendigen Dingen zusammen (Z 533:»Schmerz liegt so in unserem Leben drin, hat solche Zusammenhänge ") und nicht mit einem verborgenen Bereich von inneren Zuständen und Prozessen. Das habe ich oben in der Einleitung gemeint, wenn ich gesagt habe, dass wir die Antwort auf die Frage nach dem Wesen der Seele am falschen Ort suchen, wenn wir unser sinneres Auge auf das richten, was verborgen in unserem Innen geschehen muss, statt hinzuschauen und zu sehen, wie unser gemeinsames Leben mit den andern den wesentlichen Zusammenhang unserer psychologischen Begriffe darstellt.

Der Begriff `Schmerz beschreibt also nicht ein Etwas, das irgendwie verborgen innerhalb des Körpers ist. Aber die Empfindung Schmerz wird damit bei Wittgenstein auch nicht zu einem Nichts (vgl. PU 304), sondern indem er unseren Blick auf die grammatischen Zusammenhänge von `Schmerz` innerhalb

"our sharing routes of interest and feeling, modes of response, senses of humor and of significance and of fulfillment, of what is outrageous, of what is similiar to what else, what a rebuke, what forgiveness, of when an utterance is an assertation, when an appeal, when an explanation - all the whirl of organism Wittgenstein calls sforms of life« (Cavell 1976, 52) 


\section{Andreas Hunziker}

lenkt, macht er uns auf die lebensweltliche Bedeutung aufmerksam, welche dieses Phänomen für uns in unserem alltäglichen Leben hat. Und dazu gehört schliesslich auch dies:

"Ist es der Körper, der Schmerzen fühlt? - Wie ist dies zu entscheiden? Wie macht es sich geltend, daß es nicht der Körper ist? - Nun, etwa so:Wenn Einer in der Hand Schmerzen hat, so sagt's die Hand nicht (außer sie schreibt's), und man spricht nicht der Hand Trost zu, sondern dem Leidenden; man sieht ihm in die Augen.

Wie bin ich vom Mitleid für diesen Menschen erfüllt? Wie zeigt es sich, welches Objekt das Mitleid hat?« (PU 286f).

Es zeigt sich hier noch einmal, was es nach Wittgenstein heisst, nicht-reduktiv und nicht-metaphysisch zugleich über das Seelische zu sprechen: Durch die grammatische Betrachtung werden wir uns bewusst, dass wir in unserem alltäglichen Umgang miteinander Schmerz weder einem abstrakten Aussen noch einem abstrakten Innen zuschreiben, sondern dem Menschen, der Schmerzen hat. Und PU 287 macht darauf aufmerksam, dass diese `Zuschreibung und damit das sogenannte Problem des Geistes des Anderen nicht primär ein epistemologisches, sondern ein praktisch-existenzielles Problem darstellt: In meiner Einstellung zum anderen als Einstellung zur Seele geht es weniger um ein kognitives Problem, als um die praktischexistenzielle Frage, ob ich den andern als Seele anerkenne oder vermeide - konkret: ob ich auf den Schmerz des anderen mit Mitleid oder mit Indifferenz, Schadenfreude usw. reagiere. Stanley Cavell spricht in diesem Zusammenhang von dem vom Skeptiker und vom Anti-Skeptiker geteilten Bedürfnis, vor dem Gewöhnlichen zu fliehen und der damit verbundenen Tendenz, unsere menschliche Endlichkeit und Getrenntheit von den andern (und von der Welt und von uns selbst) in ein intellektuelles Problem zu transformieren. Cavell konfrontiert dem das, was er die (indirekte) , Wahrheit des Skeptizismus nennt: Unser gewöhnliches Verhältnis zu den anderen (und zur Welt und zu uns selbst) ist nicht primär kognitiv-erkennender (epistemologischer), sondern expressiver und anerkennender (praktisch-existenzieller) Natur:

"The mythology according to which the body is a picture implies that the soul may be hidden not because the body essentially conceals it but because it essentially reveals it. The soul may be invisible to us the way something absolutely present may be invisible to us.... But the idea of the body plays its role. In the fantasy of it as veiling, it is what comes between my mind and the other's, it is the thing that separates us. The truth here is that we are separate, but not necessarily separated (by something); that we are, each of us, bodies, i.e., embodied; each is this one and not that, each here and not there, each now and not then. If something separates us, co- 
mes between us, that can only be a particular aspect or stance of the mind itself, a particular way in which we relate, or are related (by birth, by law, by force, in love) to one another - our positions, our attitudes, with reference to one another" (Cavell 1979, 369).

\section{Glaube mit Seele}

Ich schliesse meine Überlegungen mit drei Hinweisen, inwiefern Wittgensteins Philosophie der menschlichen Seele auch für die neu erwachte Diskussion um den Seelenbegriff in der Theologie interessant sein könnte.

- Eine Untermauerung von Großhans' Argumentation müsste zeigen, dass Wittgensteins Philosophie des Gewöhnlichen nicht nur eine (negative) Kritik der Denkform Subjektivität, sondern auch eine (positive) Alternative zu dieser Denkform darstellt und damit die particula veri der Denkform Subjektivität zu integrieren vermag. $\mathrm{Zu}$ einem überzeugenden Durcharbeiten der metaphysischen Phantasien des modernen Selbstverständnisses des Protestantismus braucht es beides. Wittgensteins philosophische Psychologie (nicht zuletzt in ihrer Weiterführung durch Cavell) scheint mir dies zu leisten. Ist damit aber das von seinem sprachlichen Ausdruck und seiner reflexiven Gestalt unterschiedene subjektive religiöse Bewusstsein in Frage gestellt, dann verliert nicht nur die im Neuprotestantismus dominierende Nachordnung der fides quae gegenüber der fides $q u a$, sondern auch die neuprotestantische Innerlichkeitsemphase an Plausibilität.

- Ulrich Barth versteht seinen Aufsatz im Sinne von Vorbemerkungen zur »Beantwortung der Frage, inwieweit seelische Vorgänge sich im Aufbau von Religion zur Geltung bringen oder umgekehrt religiöse Erfahrungen das emotionelle Innenleben des Menschen formen und stabilisieren" (Barth 2004, 198). Wer dieses Interesse an der Wirklichkeit des Glaubens teilt und seine Begrifflichkeit philosophisch reflektieren will, dies aber weder auf eine metaphysische noch eine szientistische Weise tun will, für den könnte Wittgensteins Philosophie der Psychologie eine interessante Alternative darstellen.

Der Einbezug der Psychologie ist damit nicht mehr einseitig auf die psychologische Fundierung des Glaubens ausgerichtet, sondern dient vielmehr der Untersuchung der mannigfaltigen Weisen, wie die Bereiche Glaube und Seele ineinander greifen, ineinander greifen könnten und ineinander greifen sollten. Dass 'wir mit Gott` sind und damit auch, dass >Gott mit uns ist, lässt sich nicht irgendwie aus der philosophischen Besinnung auf die menschliche Seele ableiten. Aber diese Besinnung hilft zu besser zu verstehen, wie dieses /wir mit Gott`aussehen könnte, wenn wir es vom `Gott mit uns her zu verstehen, zu leben versuchen. 


\section{Andreas Hunziker}

Dies scheint mir gegenüber Ulrich Barths Vorschlag in zweifacher Weise überzeugender zu sein: Problematisch sind ja nicht nur die hinter dessen Konzeption stehenden Begründungsphantasien, sondern als ebenso unbefriedigend hat sich herausgestellt, wie rabstrakt seine Darstellungen des Seelischen geblieben sind - es würde mich wundern, wenn sich diese Abstraktheit nicht auch in Barths Beantwortung der Frage, wie seelische Vorgänge sich im Aufbau von Religion zur Geltung bringen oder umgekehrt religiöse Erfahrungen das emotionelle Innenleben des Menschen formen und stabilisieren, reflektieren würde.

- Wittgenstein sagt in PU II, xiv, 580:

"Die Verwirrung und Öde der Psychologie ist nicht damit zu erklären, daß sie eine junge Wissenschaft sei; ihr Zustand ist mit dem Physik z.B. in ihrer Frühzeit nicht zu vergleichen. ... Es bestehen nämlich, in der Psychologie, experimentelle Methoden und Begriffsverwirrung....

Das Bestehen der experimentellen Methode läßt uns glauben, wir hätten das Mittel, die Probleme, die uns beunruhigen, loszuwerden; obgleich Problem und Methode windschief aneinander vorbeilaufen«.

Wittgensteins begriffliche Untersuchungsmethode zu den Grundlagen der Psychologie bietet ein sehr sensibles Instrumentarium, damit die Theologie auch mit der empirisch verfahrenden Psychologie und deren Seelenverständnis in ein produktiv-kritisches Gespräch treten kann. Ohne diese Arbeit am Begriff wird es dem theologischen Denken schwer fallen, sich in diesem Gespräch nicht entweder in den Begriffsrahmen der empirischen Psychologie (samt deren begrifflichen Verwirrungen) einzuordnen oder einfach diejenigen ıneuesten Resultate der Psychologie an das eigene Seelenverständnis anzufügen, die dazu gerade am besten zu passen scheinen.

\section{Literatur}

Barth, U. (2004), Selbstbewußtsein und Seele, ZThK 101, 198-217.

Cavell, St. (1969), The Availability of Wittgenstein's Later Philosophy, in: ders., Must We Mean What We Say?, Cambridge, 44-72.

Ders. (1979), The Claim of Reason. Wittgenstein, Skepticism, Morality, and Tragedy, Oxford/New York.

Großhans, H.-P. (2005), Das moderne Selbstverständnis des Protestantismus und Wittgensteins Kritik der Subjektivität, in: Dalferth, I.U./Stoellger, Ph. (Hg.), Krisen der Subjektivität. Problemfelder eines strittigen Paradigmas, 63-77 (im Erscheinen). 
McGinn, M. (1997), Wittgenstein and the Philosophical Investigations, London u.a.

Ders. (1993), Review to „Ludwig Wittgenstein, Last Writings on the Philosophy of Psychology, Volume 2: The Inner and the Outer", Mind, New Series, Vol. 102, Issue 408, 697-701.

Schnädelbach, H. (2001), Phänomenologie und Sprachanalyse, in: Wingert, L./Günther, K. (Hg.), Die Öffentlichkeit der Vernunft und die Vernunft der Öffentlichkeit, Frankfurt a.M., 243-267.

Wittgenstein, L., Das Blaue Buch, in: Werkausgabe Bd. 5, Frankfurt a.M. 71997, 15-116.

Ders., Philosophische Untersuchungen, Frankfurt a.M. 2003.

Ders., Philosophische Untersuchungen II. In: Werkausgabe Bd. 1, Frankfurt a.M. 91993, 487-580.

Ders., Zettel, in:Werkausgabe Bd. 8, Frankfurt a.M. 61994, 259-443.

- Andreas Hunziker ist Assistent am Lehrstuhl von Prof. Dr. I.U. Dalferth an der Universität Zürich. 